\title{
Cutting-Edge Life Sciences in Swiss Gymnasia - Success is not a Secret
}

\author{
Thomas P. Werner and Samuel S. Ginsburg*
}

\begin{abstract}
What can be done to achieve a high-quality education in Life Sciences at gymnasia level that arouses the interest of most students and motivates them to accomplish high performances? The recipe for success is simple: Employ excellent natural scientists who combine the passion for their subject with strong pedagogic skills as teachers to your school. The challenge is to create an environment that makes teaching attractive for scientists and that has a positive and lasting effect on the motivation of the teachers. We consider the following criteria especially significant: The social structure/arrangement is likely to be the key aspect. Ideally it consists of a constructive relation between the teachers, the school administrators, the staff and most importantly the students. The work must be challenging, but the workload should be in a good proportion to the resources of the teachers. To communicate a realistic impression of Life Sciences an adequate infrastructure and facilities are necessary that allow an education based on experiments and practica. The curriculum should consider the interests of the students and should contain topics which are relevant for society. Finally it should grant enough educational latitude for the teachers so they can use their specialized skills. Contacts to other gymnasia, universities and industry are important for orientation, the exchange of knowledge and to enable extracurricular projects.
\end{abstract}

Keywords: Education · Gymnasia · Instructor · Life sciences · Teacher

\section{Excellent Teachers}

The central premise for a lasting and exciting development of Life Sciences at our school is to employ the most dedicated and enthusiastic biologists who are pedagogically talented and like to work with adolescents and peers. To accomplish this challenge a position at our school has to be at least as attractive and fascinating for a scientist as a career in the industry or at a university/academy.

But what is the meaning of 'pedagogically talented'? In our opinion the following qualities are of vital importance: First, a qualified teacher has sympathy for the students. The teacher confides in the students, listens to them and takes them seriously. Students associate the subject with the instructor. If the latter is unappealing
${ }^{*}$ Correspondence: Dr. S. S. Ginsburg Kantonsschule Wettingen

Klosterstrasse 11

$\mathrm{CH}-5430$ Wettingen

Tel.: +41448445610

E-mail: gis@kswe.ch

http://www.kanti-wettingen.ch/ the subject will tend to be perceived as unalluring. Secondly, the teacher should fascinate the students with his subject thereby evoking interest and curiosity. Thirdly, the instructor has to communicate his expectations clearly and to keep them on an accomplishable level for most students. This way most students may be successful and success is the most important factor to reinforce motivation and self-confidence.

The next key issue is: What makes a gymnasium attractive for a scientist who fulfills those criteria? We consider two aspects especially significant: The social arrangement at the working environment and a contemporary infrastructure that allows diversified education that is based on experiments.

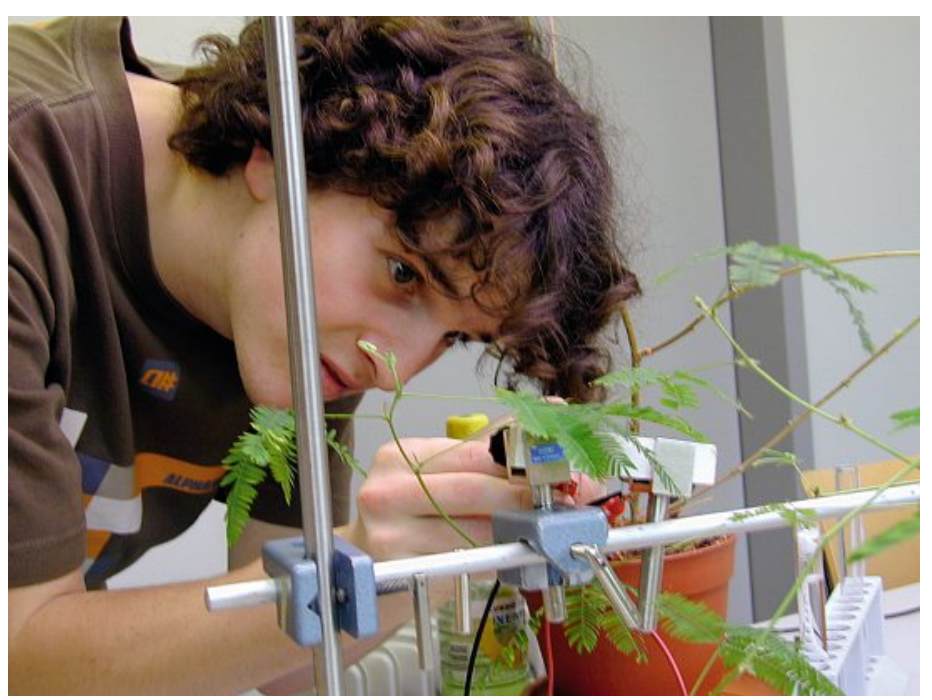

\section{The Social Arrangement}

The same is true for instructors, students, school administration and all human resources: People like to work if they are accepted and their work is appreciated and if they are neither under challenged nor unable to cope with the workload. A permanent exchange of ideas and experiences between instructors and active support from technicians are necessary for a diversified education of high quality. This way approved elements can be passed down to other instructors and at the same time new concepts can be established. The collaboration with the school administration is equally important. It is the best premise for work, if both sides confide in and ap- 


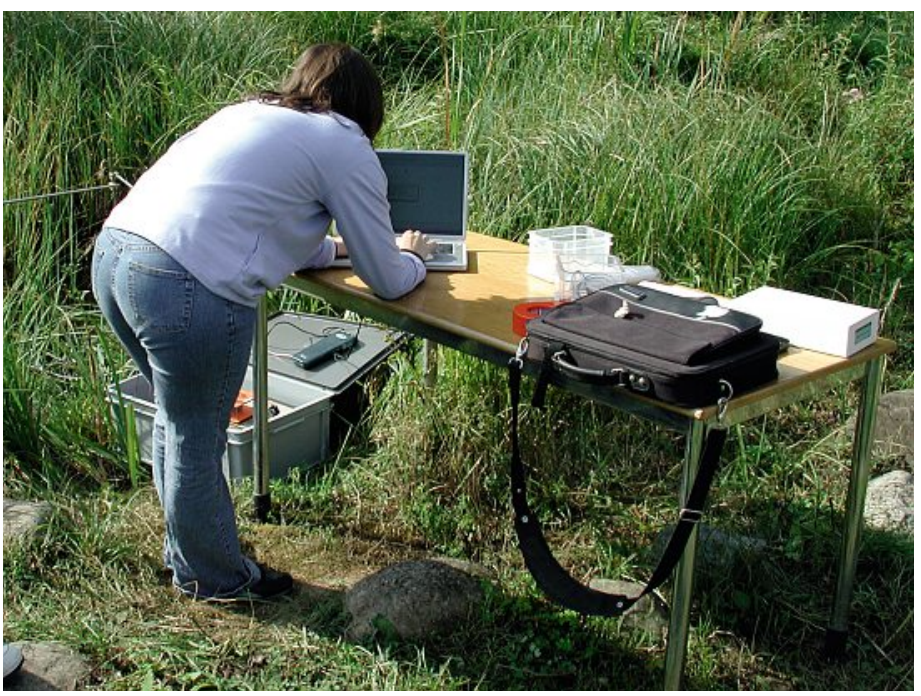

Measuring different parameters in the school pond over 24 hours.

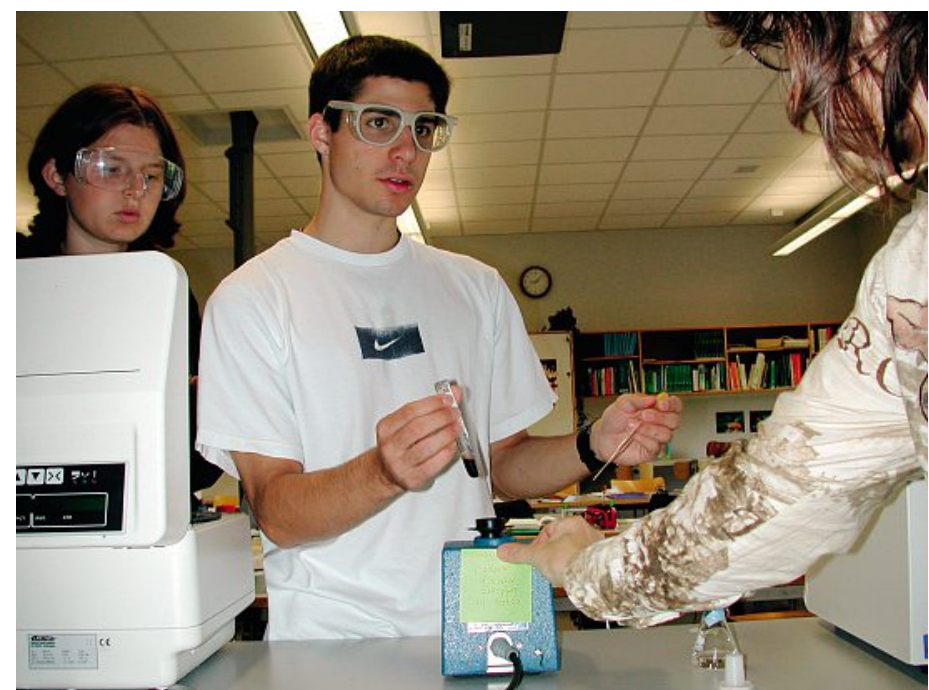

Extracting chromosomes and analyzing the karyogram. preciate each other. Teachers with a strong attitude like to study further, get involved with work groups and contribute in this way to an animated and rich school culture.

\section{Infrastructure}

As developments in Life Sciences are growing constantly and very fast it is clear that in order to keep up with modern technologies not only modern biology has to be taught but also the state of the art technologies as they are used in universities and companies nowadays have to be taken into consideration in today's teaching and the students have to know how to handle these tools. With this knowledge the students are also enabled to take part in today's discussions of crucial questions in social life about new technologies and are accurate in discussing it. They also get an insight of what Life Science studies might be about or what jobs and careers in this field may offer them.

Even if the basis for a positive connotation of natural sciences is already set at kindergarten level and most of the time also through behavior of the parents, personal experiences during formation at high school are of great importance. Direct contact with objects of research and handson experiments make teaching closer to nature and creates deeper impressions as theoretical exercises. With these practical work settings a concrete image of how scientific studies are performed nowadays can be provided. This kind of teaching method enhances the skills of observation and unwind logical thinking - all of these are skills that are crucial to becoming a future scientist and are helpful for any other type of job.

\section{Syllabus}

Teachers must feel comfortable with the curriculum they have to follow, ideally by personal identification. Not only a didactically well elaborated structure of the basic knowledge, the definition of the educational objective and learning targets, the options for many different experimental skills are of importance. The syllabus must offer a certain degree of freedom to enable teachers to deploy their excellence. This freedom is inalienable if we want to have teachers exploiting their full abilities.

In addition the syllabus should give rise to discussions of social interest. To discuss with pupils of topics they are especially interested in is an important issue if we aim to work with students who try to get to the bottom of open questions instead of training believers.

It makes sense to integrate all available options that a school can offer into the syllabus to encourage teachers to provide an exciting and pulsating education.

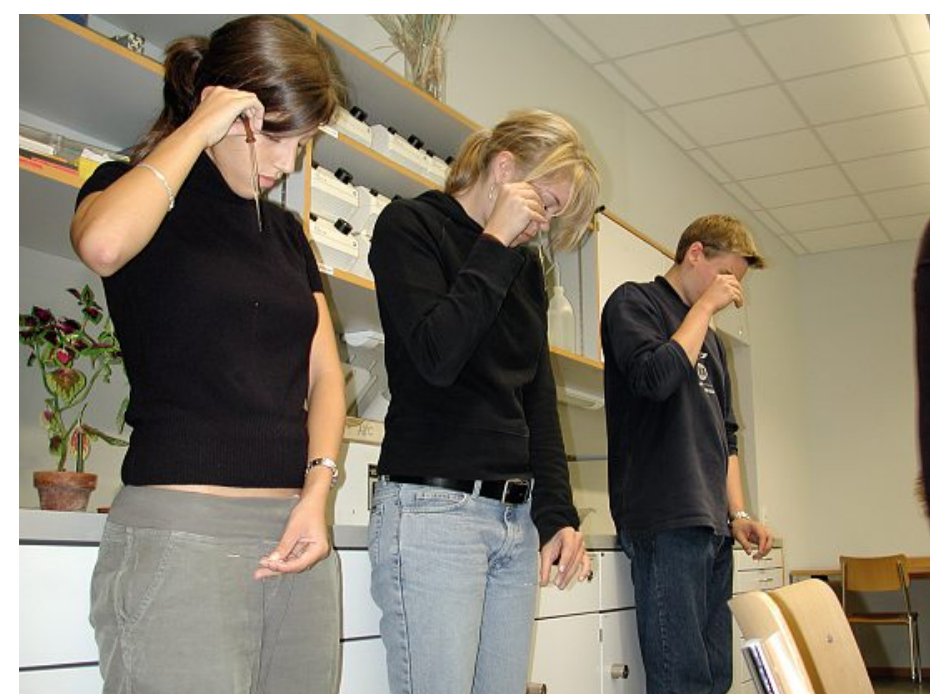

\section{Teaching Material}

If the textbook offers a lot of training opportunities and pupils feel comfortable with the presentation of the content, there is a good chance that most of the theory can be prepared at home. Therefore comprehensibility is of crucial importance. The content must conform to the current level of knowledge and most of the subject areas of the syllabus should be covered. The international edition of the textbook 'Biology' by N. Campbell ('Biology', N. Campbell, 9th edition (Global Edition), Pearson, 2011) matches perfectly, at least at AP-level (Schwerpunktfach).

During lessons the acquired content may be discussed and there remains enough time to concentrate on experiments. The well-elaborated system of the 'Mastering Biology' web program allows the teacher to support students individually. For all pupils who will continue studying at the university or the ETH it is very helpful to be familiar with this learning strategy - they will use the same textbook during the first two semesters.

Hands-on activity with leucocytes. 


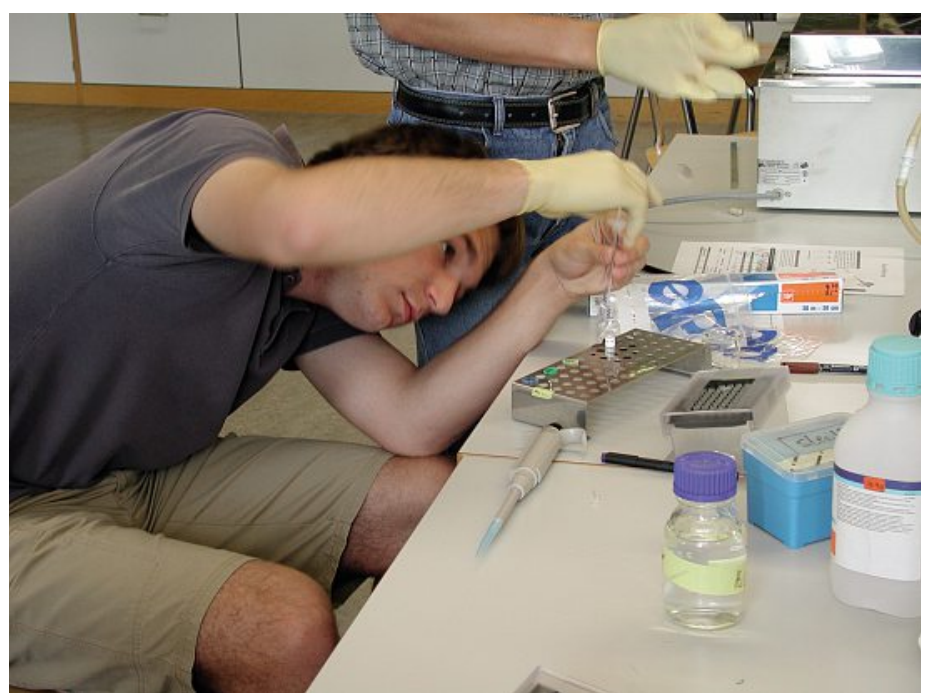

Handling small volumes for the first time.

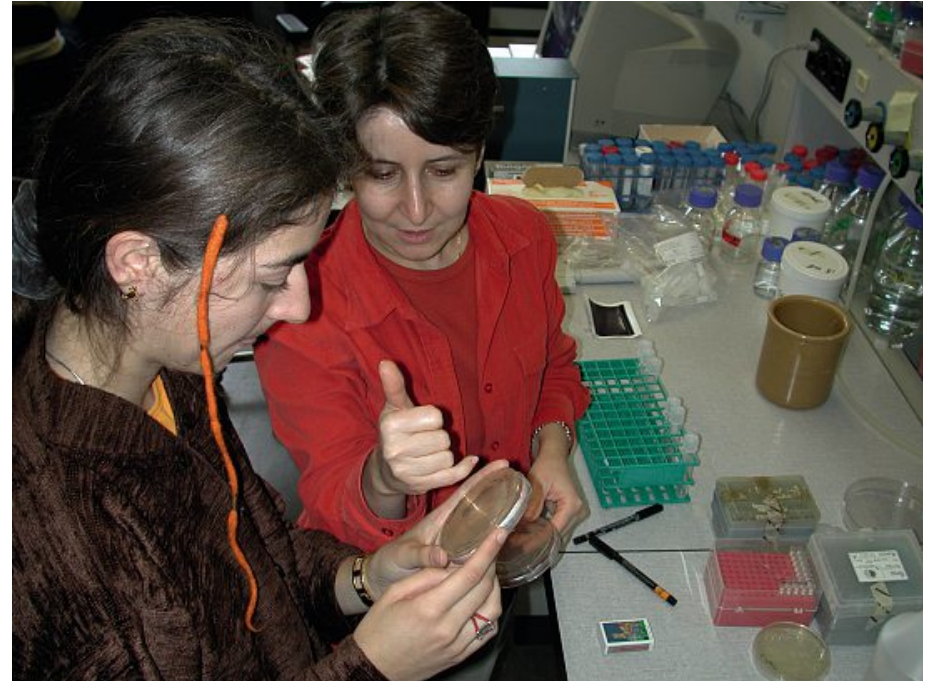

Discussing results of a molecular genetic investigation at a university lab.

\section{Extrascholar Contacts}

While as in former times gymnasia were mostly criticized as 'ivory towers', nowadays successful high schools have realized that 'open doors' have many advantages. Contact and an active cooperation with universities and the industry are a win-win situation for them as well as for the teachers and the students.

At Wettingen Kantonsschule we maintain close contacts to other institutions in Switzerland and abroad. We offer for example a three-week practical training at ETH and the University of Zurich where the students may gain practice and work for their maturity work. Practical training is sometimes also offered by industry.

Scientists from universities are invited to our gymnasium and present their work, to give an authentic insight in their research and work-field. The industry also helps to organize and finance a yearly Life Science Symposium where teachers from Germany, France, Switzerland and the US gather for a day of high-level exchange of knowledge and performing of workshops. During the so-called 'Bridge-Projects' which are organized and financed by Interpharma, students may experience a day or more in a lab of the University of Bern and find out what it means to be a researcher, after having learnt the basic skills at school. These kind of contacts lead to a close personal relationship between the teachers and the institutions. Out of these fruitful contacts often new projects grow. This also helps to prevent prejudice and avoid the fear of contact between partners. At the same time it is becoming obvious that finally everybody profits from these relationships and that the main goal is to fascinate young people for Life Sciences. For many years the teachers from Gymnasium of Wettingen are obtaining further education by orga- nizing the 'MicroEvents'. These events are very low threshold and are organized in one of the six high schools in order to exchange teaching material from a set part of the syllabus. These events are especially rewarding for young teachers that are new in the teaching field. Through exchanging of the so-called 'trouvailles' (this may be an excellent experiment, a hands-on activity, good documentations, movies etc.), everybody profits from 'best practice' of the others in a peer-to-peer situation. At the same time theses micro-events fulfill social criteria like getting to know each other and so on. Being part of an active group of science teachers helps to prevent the feeling of being a 'lone warrior'.

\section{Public Private Partnership}

Experimental work in Life Sciences is expensive. Equipment and chemicals, especially kits are costly. Successful applications have to be clearly declared and elucidated. If private partners and representatives of the public government collaborate because they recognize an idea as valuable, even bigger projects can be realized under the premise that students will profit substantially and the whole biology department becomes more attractive. But, all the investments are worthless if there are no teachers willing to invest time and energy to embed the new technologies in the curriculum. It is important to discuss the new approaches with colleagues to get a long lasting change also because incorporation, the initial skill adaption training is demanding and deserves continuity. Finally it is important to keep all partners in touch and to prove that the investment was a success.

\section{Extracurricular Activities}

To be involved in a national or international competition is exciting and stimulating for pupils. Teachers get an impression about the state of the art of their education. Some examples of extracurricular activities are: National and International Biology Olympiads, SJF Projects (Schweizer Jugend forscht), Science on the Move (SimplyScience), ISSI (Weizmann Institute, Israel), College Day (Biovalley College Network BCN), Schülerkongress (BCN) and further more. Our pupils regularly take part in these events and thereby gain self-confidence.

In the context of the Biovalley College Day pupils can win awards for outstanding maturity works and share their experience with colleagues from Germany and France. Meanwhile they recognize how important networking in science is. Pupils learn how to communicate in a foreign language and gain familiarity with new cultures.

\section{Conclusion}

The most important factor in the diverse areas of the management of a high school are the teachers. The person who is in charge of employing new teachers is very crucial and therefore carries a great responsibility. Mostly the Head of Schools recruits the new teachers like in our case in a constructive cooperation with the Head of Department.

A head of Schools who leads his/her team carefully and shares responsibility with the teachers creates a good atmosphere. This is the fundament for a successful Gymnasium in which all the partners are committed and everybody is willing to contribute above the norm. 